\title{
High-speed side-shooter using Leidenfrost phenomena
}

Cite as: J. Appl. Phys. 125, 134502 (2019); https://doi.org/10.1063/1.5064429

Submitted: 04 October 2018 . Accepted: 15 March 2019. Published Online: 04 April 2019

Hideyuki Sugioka (D), Satoru Segawa, and Mako Kubota

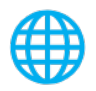

\section{Applied Physics Reviews} Now accepting original research 


\title{
High-speed side-shooter using Leidenfrost phenomena
}

\author{
Cite as: J. Appl. Phys. 125, 134502 (2019); doi: 10.1063/1.5064429 \\ Submitted: 4 October 2018 . Accepted: 15 March 2019. \\ Published Online: 4 April 2019
}

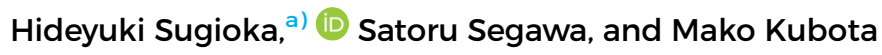

\author{
AFFILIATIONS \\ Department of Mechanical Systems Engineering, Shinshu University, 4-17-1 Wakasato, Nagano 380-8553, Japan

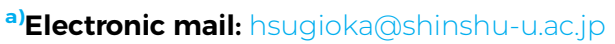

\begin{abstract}
Liquid delivery systems are important in microfluidic applications. In this paper, we report a high-speed side-shooter using a simple platelike hot protrusion placed on the lower edge of a hot slope. In particular, we experimentally show that the side-shooter launches a droplet with a high speed $(\sim 15 \mathrm{~cm} / \mathrm{s})$. We propose a simple model to explain the side-shooting phenomenon; i.e., the hot protrusion causes an increase in the surface energy due to the formation of a new folded surface, which results from a new Leidenfrost phenomenon coupled with the protrusion, and therefore, the droplet can be launched at a high speed on the hot slope with a low friction due to the ordinary Leidenfrost phenomenon. A comparison of the theoretical results with the experimental results shows that they are in good agreement with each other. We believe that our side-shooter will play an important role in microfluidics in the future.
\end{abstract}

\section{Published under license by AIP Publishing. https://doi.org/10.1063/1.5064429}

\section{INTRODUCTION}

The development of miniaturized high speed liquid delivery systems is essential for integrated microfluidic systems such as lab-on-a-chip and micrototal analysis systems ( $\mu$ TAS). ${ }^{1}$ Therefore, various studies have been devoted to this purpose, ranging from the classical dc electro-osmotic pumps ${ }^{2}$ to the challenging liquid delivery systems. $^{3-5}$ Nevertheless, the typical flow velocity of a dc-electro-osmotic (DCEO) pump is still in the order of $0.1 \mathrm{~mm} / \mathrm{s}$, and even the improved ac-electro-osmotic (ACEO) pump just produces a flow velocity of the order of $1 \mathrm{~mm} / \mathrm{s}^{3}$ Although electrowetting actuators have demonstrated average velocities of $3 \mathrm{~cm} / \mathrm{s}$ by using the electro-wetting phenomenon, ${ }^{4}$ they require high voltages (e.g., 40-80 V) with a corresponding matrix driving system, which may increase the cost of the system significantly. Linke et al. reported the self-propelled motion of a droplet at $\sim 5 \mathrm{~cm} / \mathrm{s}$ on hot surfaces with an asymmetric ratchet-like topology over the Leidenfrost temperature. This has attracted considerable attention, and extensive studies ranging from fundamental studies to application research have been conducted on this topic.

For example, Ok et al. ${ }^{7}$ reported that submicron Leidenfrost ratchets yield water droplet velocities of up to $\sim 40 \mathrm{~cm} / \mathrm{s}$. Cousins et al. reported a trapping phenomenon using a concentric Leidenfrost ratchet. ${ }^{8}$ Furthermore, with regard to the mechanism, Wüger showed the possibility of thermal creep by performing an analytical evaluation of the temperature distribution. ${ }^{9}$ By using the Boltzmann equation, Hardt et al. showed that the dominant factor is the pressure-driven flow. ${ }^{10}$ By using the lattice Boltzmann modeling, Li et al. clearly showed that the motion of self-propelled Leidenfrost droplets is due to the asymmetry of the ratchets and the vapor flows beneath the droplets. ${ }^{11}$ It should be noted that the Leidenfrost phenomenon is a kind of film boiling phenomena of a droplet on a hot plane substrate. Furthermore, it is characterized by the hovering state of the droplet and the long lifetime of the droplet over the Leidenfrost temperature $T_{L}$; e.g., $T_{L} \sim 150^{\circ} \mathrm{C}$ for a water droplet of diameter $1 \mathrm{~mm}$ on a duralumin plate. ${ }^{12}$ These two characteristics result from the existence of a vapor layer between the droplet and the substrate due to the evaporation of the droplet and the balance of the heat conduction from the substrate to the droplet. ${ }^{12}$ Obviously, Leidenfrost phenomena have much potential to achieve a high-speed liquid delivery system, which cannot be achieved by other phenomena.

In particular, new devices using Leidenfrost phenomena are promising, ${ }^{6}$ and innovative studies are being devoted to this problem even now. For example, from the viewpoint of world-wide energy reduction and microfluidic applications, Hashmi et al. demonstrated that a small cart on a liquid can be transported at a high speed $(\sim 16 \mathrm{~cm} / \mathrm{s})$ along the hot ratchet surface by utilizing a Leidenfrost phenomenon. ${ }^{13}$ Wells et al. demonstrated a sublimation 
heat engine that works under ultralow friction conditions, inspired by the self-propelled Leidenfrost phenomenon. ${ }^{14}$ Shi et al. ${ }^{15} \mathrm{dem}-$ onstrated a self-propelled hovercraft based on a cold Leidenfrost phenomenon and suggested that it could be used as a cargo ship or a petroleum contamination collector. Sugioka and Segawa ${ }^{16}$ proposed a controllable Leidenfrost glider on a shallow water layer and demonstrated that the vehicle can move at a velocity of $\sim 20 \mathrm{~cm} / \mathrm{s}$. Arter et $a l^{17}$ reported the conditions under which the droplet velocity can exceed $35 \mathrm{~cm} / \mathrm{s}$ by using a single device with a continuously adjustable ratchet surface. Furthermore, as pointed out by Quéré, controlling ultramobile Leidenfrost drops is one of the main future directions, ${ }^{6}$ and from this viewpoint, Luo et al. explored selfpropulsion of a Leidenfrost drop between nonparallel structures. ${ }^{18}$ In addition, new experimental facts, which suggest the possibility of new self-propelling devices, have been discovered recently. For example, Minal et al. ${ }^{19}$ found that a drop on a hot ratchet has a combined motion of translation and self-rotation. Pham et al. ${ }^{20}$ observed that hydrogel drops, initially at rest on a surface, spontaneously jump upon rapid heating and continue to bounce with increasing amplitudes. Bouillant et $a l^{21}$ reported that Leidenfrost droplets initially at rest on horizontal substrates self-rotate and selfpropel in the direction they are rolling.

However, the fundamental design principle for future devices using Leidenfrost phenomena is still unclear. Furthermore, a high speed side-shooter using a hot protrusion has not been explored so far. Therefore, in this study, we propose a high-speed side-shooter based on hot protrusion and clarify its mechanism, which may provide a design principle for future devices with promising performance. Namely, in this study, we focus on a side-shooter based on hot protrusion and experimentally prove that it can shoot a droplet at a high speed $(\sim 0.15 \mathrm{~m} / \mathrm{s})$. Furthermore, we propose a simple self-consistent model that explains the side-shooting phenomenon, which is caused by the formation of a new folding surface due to a new Leidenfrost phenomenon, along with the hot protrusion, and discuss the mechanism.

\section{EXPERIMENTAL METHOD}

Figure 1(a) shows the proposed side-shooting system (using a Leidenfrost phenomenon), which consists of an oblique hot brass plate with an inclination angle $\theta\left(=3^{\circ}\right)$ at temperature $T_{s, 1}\left(\simeq T_{s}\right)$, a hot protrusion brass plate (placed on the lower edge of the hot slope at the position $X=0)$ at $T_{s, 2}\left(\simeq T_{s}\right)$, and a hemisphere droplet of water with a radius $r_{0}(=3.15 \mathrm{~mm})$. More precisely, the brass plate of thickness $1 \mathrm{~mm}$ is placed on the surface of a laboratory hot plate, and the brass plate is heated to $T_{s}$ by the laboratory hot plate; i.e., the temperature $T_{s}\left(=300^{\circ} \mathrm{C}\right)$ is measured on the surface of the laboratory hot plate. In detail, $T_{s, 2}\left(=298^{\circ} \mathrm{C}\right)$ was measured on the surface of the root of the protrusion by using a thermocouple thermometer in our experiment. Therefore, the relation $T_{s} \simeq T_{s, 1} \simeq T_{s, 2}$ is justified within an accuracy of $2{ }^{\circ} \mathrm{C}$; i.e., there is no large difference among $T_{s}, T_{s, 1}$, and $T_{s, 2}$, although Auliano et ll $^{22}$ reported that there can be a large difference of about $95^{\circ} \mathrm{C}$ between the temperature measured on the surface and the temperature measured in the heating block. Furthermore, by placing the droplet on the slope at $X=X_{0}$, we observed that the droplet slid down toward the hot protrusion with a very low friction force $\left(f_{v} \sim 0 \mathrm{~N}\right)$ compared with the tangential projection of the droplet weight due to the ordinary Leidenfrost phenomenon of the hot slope, and after contacting the protrusion, the droplet was launched to the upper position at $X=X_{1}$ because of the Leidenfrost phenomenon coupled with the new surface formation that accumulated the surface energy due to the surface tension $\gamma=58.9 \mathrm{mN} / \mathrm{m}$ at the boiling temperature $T_{b}\left(=100^{\circ} \mathrm{C}\right)$ of water. Here, we used deionized water (milli-Q, $18.2 \mathrm{M} \Omega \mathrm{cm}$ ). Furthermore, we determined the experimental dependence of the position $X(t)$ on $t$ from the video data of size $1280 \times 720$ and frame rate of $240 \mathrm{fps}$. Specifically, we analyzed the motions of the droplet numerically by using the video data with an image processing technique; i.e., by performing binarization of the video data with a suitable threshold, we detected the droplet pixels and determined the position $X(t)$ of the center of gravity.

Figure 1(b) shows the detailed structure of the protrusion [gap $l_{1}(=1 \mathrm{~mm})$, thickness $l_{2}(=1 \mathrm{~mm})$, and depth $\left.l_{3}(=2.5 \mathrm{~mm})\right]$ just before the collision of the droplet with the protrusion at $t=t_{0}$, while Fig. 1(c) shows the deformation of the droplet during the surface formation process of the new Leidenfrost phenomenon. From the video data of the experiment, we determined the maximum push-in widths $w_{1}$ and $w_{2}$ at the lower and higher positions, respectively, the average push-in width $w_{b}=\left(w_{1}+w_{2}\right) / 2$, the corresponding times $t_{w 1}$ and $t_{w 2}$, and the average push-in time $t_{b}=\left(t_{w 1}+t_{w 2}\right) / 2$. Furthermore, we define the corresponding push-in time periods as $\Delta t_{w 10}=t_{w 1}-t_{0}, \Delta t_{w 11}=t_{1}-t_{w 1}, \Delta t_{w 20}=$ $t_{w 2}-t_{0}, \Delta t_{w 21}=t_{1}-t_{w 2}, \Delta t_{w b 0}=t_{w b}-t_{0}$, and $\Delta t_{w b 1}=t_{1}-t_{w b}$. In order to observe the surface formation process in detail, we set a video camera having a wide-angle lens in front of the hot protrusion of the side shooter. Since there was a distortion at the edge area of the image, we corrected the position $X$ as $X\left(X^{\prime}\right)=h_{s}\left(X^{\prime}\right) X^{\prime}$, where $h_{s}\left(X^{\prime}\right)=1+\left(h_{s 0}-1\right) X^{\prime} / X_{a}^{\prime}$ is a correction factor, $X_{a}^{\prime}=4.25 \mathrm{~cm}$, $h_{s 0}=5 / 4.25 \simeq 1.176$, and $X^{\prime}$ is the position before the correction. Furthermore, we observed that the shape of the droplet in air was spherical with a diameter of $\phi$ (typically, $5 \mathrm{~mm}$ ); therefore, we determined the average radius $r_{0}$ of the hemisphere from the relation $\frac{4}{3} \pi\left(\frac{\phi}{2}\right)^{3}=\frac{2}{3} \pi r_{0}^{3}$; i.e., $r_{0}=2^{\frac{1}{3}} \frac{\phi}{2}$. Furthermore, the mass $m_{p}$ of the droplet was determined as $m_{p}=\frac{4}{3} \pi\left(\frac{\phi}{2}\right)^{3} \rho$ (typically, $6.26 \times 10^{-5} \mathrm{~kg}$ for $\phi=5 \mathrm{~mm}$ ), where $\rho=957 \mathrm{~kg} / \mathrm{m}^{3}$ is the density of water at $T_{b}=100^{\circ} \mathrm{C}$. We repeated the same experiment $N_{t}$ times under the same condition and labeled $N=1-N_{t}$ (typically, 10) to differentiate them. Note that as explained in Ref. 12, the droplet on a Leidenfrost vapor layer is nearly spherical in a rest state, when the drop radius $\phi / 2$ is smaller than the capillary number $a$ ( $2.5 \mathrm{~mm}$ for water); i.e., a droplet of $\phi=5 \mathrm{~mm}$ is considered to be nearly spherical in a rest state. However, there exists a large vertical vibration in the droplet in our experiment, and our video observation tells us that as the first step, it should be dealt with as a hemisphere (on average); please see Figs. 2(a) and 2(d) and the explanation in Sec. III.

\section{OBSERVATION OF THE SIDE-SHOOTING PHENOMENON}

Figure 2 shows the photographs of the side-shooting motions $(N=4$ in experiment 1$)$. Here, $T_{s}=300{ }^{\circ} \mathrm{C}, \theta=3^{\circ}, \phi=5 \mathrm{~mm}$, $r_{0}=3.15 \mathrm{~mm}, \quad X_{0}=5 \mathrm{~cm}, \quad l_{1}=l_{2}=1 \mathrm{~mm}, \quad$ and $l_{3}=2.5 \mathrm{~mm}$. Furthermore, we have used almost the same conditions (except $X_{0}$ ) 


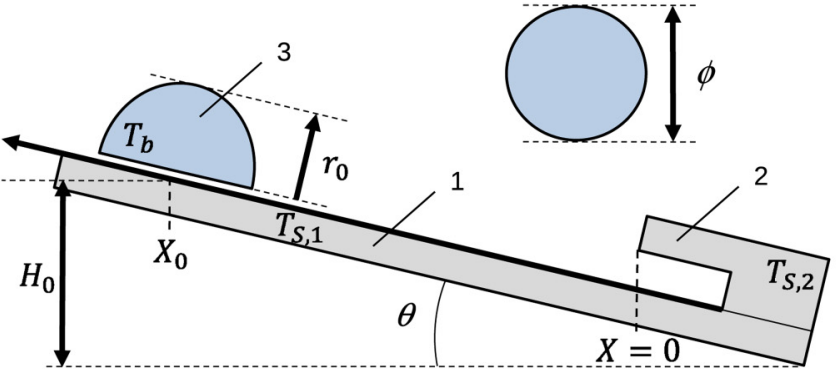

(a)

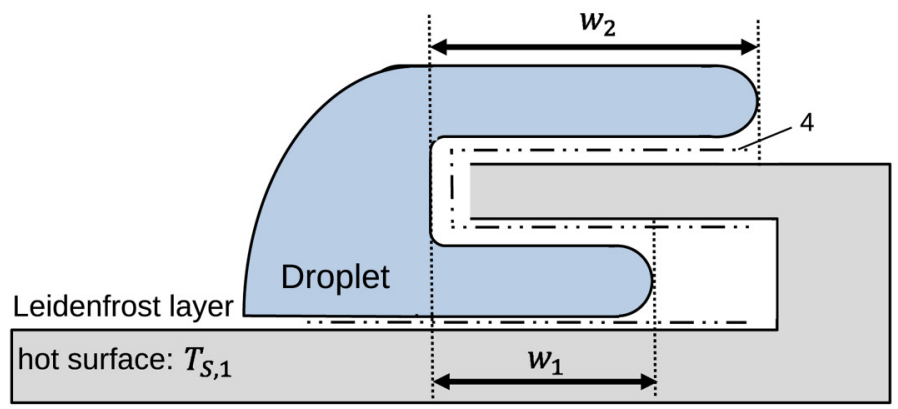

(c)

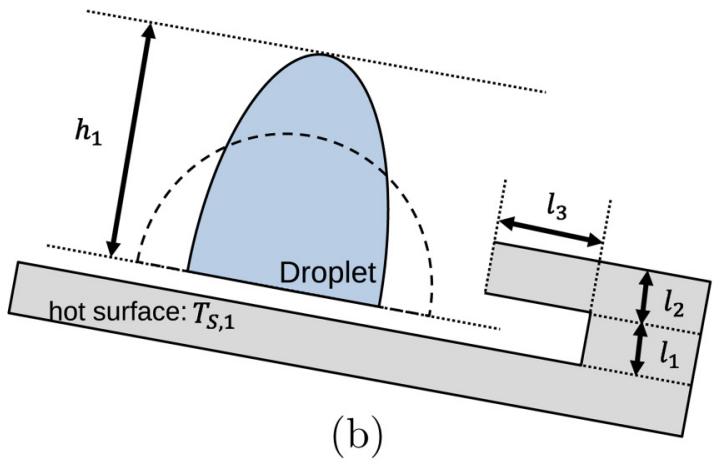

(b)

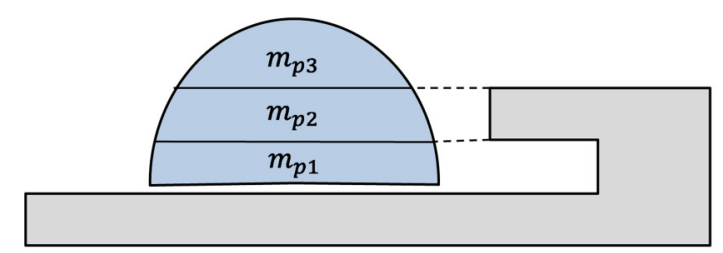

(d)

FIG. 1. Schematic view of a side-shooting system using a Leidenfrost phenomenon coupled with a protrusion. (a) Side-shooting system using a new Leidenfrost phenomenon. (b) Structure of the side-shooter using a hot protrusion plate. (c) Accumulation of surface energy due to the formation of a folded vapor layer. (d) Partial masses of the droplet for the calculation of the energy loss ratio. Here, typically, $m_{p}=6.26 \times 10^{-5} \mathrm{~kg}, \rho=957 \mathrm{~kg} / \mathrm{m}^{3}, g=9.81 \mathrm{~m} / \mathrm{s}^{2}, \gamma=58.9 \mathrm{mN} / \mathrm{m}$ at $T_{b}=100^{\circ} \mathrm{C}, T_{s} \simeq$ $T_{s, 1} \simeq T_{s, 2} \simeq 300^{\circ} \mathrm{C}$ (precisely, $T_{s}=300^{\circ} \mathrm{C}$ and $T_{s, 2}=298^{\circ} \mathrm{C}$ ), $\theta=3^{\circ}, \phi=5 \mathrm{~mm}, r_{0}=3.15 \mathrm{~mm}, X_{0}=5 \mathrm{~cm}, l_{1}=l_{2}=1 \mathrm{~mm}$, and $l_{3}=2.5 \mathrm{~mm}$. $1:$ hot slope, 2 : hot protrusion, 3 : droplet, 4 : folded vapor layer due to a new Leidenfrost phenomenon.

throughout the paper. As shown in Fig. 2(a), the droplet started to slide down from $X=X_{0}$ at $t=0$ toward the right edge of the hot slope where friction is almost zero because of the ordinary Leidenfrost phenomenon. Then, it reached the side-shooter at
$X=0$ at $t=t_{0}=0.483 \mathrm{~s}$ as shown in Fig. 2(b). The droplet was deformed at $t=t_{w 1}=t_{w 2}=0.504 \mathrm{~s}$, as shown in Fig. 2(c). Here, if the temperature of the protrusion $T_{s, 2}$ is lower than the Leidenfrost temperature $T_{L}$, the vapor layer is not generated, and therefore, a

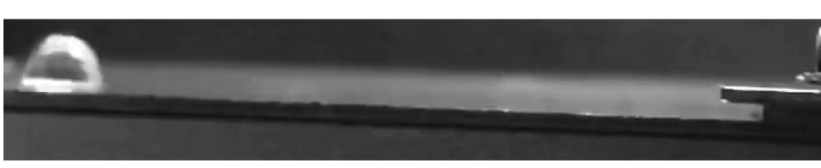

(a)

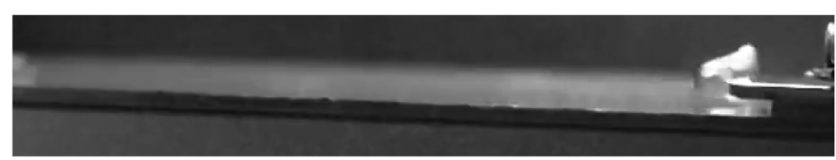

(c)

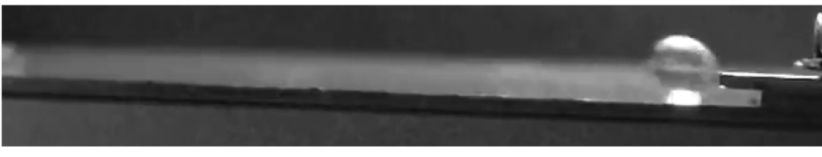

(b)

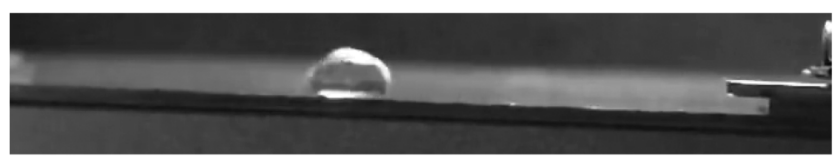

(d)

FIG. 2. Photographs of side-shooting motions ( $N=4$ in experiment 1). (a) Initial state at $X=X_{0}(t=0 \mathrm{~s})$. (b) Collision at $X=0$ ( $\left.t=t_{0}=0.483 \mathrm{~s}\right)$. (c) Deformation $\left(t=t_{w 1}=t_{w 2}=0.504 \mathrm{~s}\right)$. (d) $1 \mathrm{st}$ peak state at $X=X_{1}(t=0.883 \mathrm{~s})$. Here, $T_{s}=300^{\circ} \mathrm{C}, \theta=3^{\circ}, \phi=5 \mathrm{~mm}, r_{0}=3.15 \mathrm{~mm}, X_{0}=5 \mathrm{~cm}$, $l_{1}=l_{2}=1 \mathrm{~mm}$, and $l_{3}=2.5 \mathrm{~mm}$. 
new surface does not appear; as a result, the droplet loses the total kinetic energy, and it cannot be launched. Conversely, at $T_{s, 2}>T_{L}$, the vapor layer is generated and a new surface appears due to the Leidenfrost phenomenon. The well-known Leidenfrost phenomenon does not produce the excess surface energy that can push out the droplet in the lateral direction. Namely, we first observed that the new effect can be found only when a new folded surface is produced by the collaboration between the ordinary Leidenfrost phenomenon and the hot protrusion plate. In other words, the observed phenomenon is a new phenomenon or at least a new type of Leidenfrost phenomena.

\section{SIMPLE MODEL FOR THE SIDE-SHOOTING PHENOMENON}

\section{A. Launching performance}

To help the readers understand this concept clearly, we explain the theoretical model of the side-shooter before the experimental results. That is, we propose a simple model that explains the side-shooting phenomenon due to a new Leidenfrost phenomenon coupled with the new surface formation process. Since we can neglect the friction force between the droplet and the hot slope caused by the vapor layer due to the ordinary Leidenfrost phenomenon, the velocity of the droplet at $t=t_{0}$ is

$$
V_{0} \simeq \sqrt{2 g H_{0}}
$$

where $g=9.81 \mathrm{~m} / \mathrm{s}^{2}$ and $H_{0}\left(=X_{0} \sin \theta\right)$ is the height of the initial position. Similarly, the velocity of the droplet at $t=t_{1}$ is $V_{1} \simeq \sqrt{2 g H_{1}}$, where $H_{1}\left(=X_{1} \sin \theta\right)$ is the height of the first peak position at $X=X_{1}$. Here, $r_{\text {loss }}=E_{1} / E_{o}=\left(H_{0}-H_{1}\right) / H_{0}$ represents the energy loss ratio and $H_{1}$ is predicted as

$$
H_{1} \simeq\left(1-r_{\text {loss }}\right) H_{0} \text {. }
$$

In general, we can write that $H_{n} \simeq\left[1-r_{\text {loss }}\left(H_{n-1}\right)\right] H_{n-1}$ with $H_{n}=X_{n} \sin \theta$, where $X_{n}$ is the $n\left(=N_{p}\right)$ th peak position. Thus, if $r_{\text {loss }}\left(H_{n-1}\right) \simeq$ const., we can write $H_{n} \simeq\left(1-r_{\text {loss }}\right)^{n} H_{0}$. In this case, the corresponding maximum velocity is written as $V_{n} \simeq \sqrt{2 g\left(1-r_{\text {loss }}\right)^{n} H_{0}}$. Note that $r_{\text {loss }}$ is constant at $l_{3} \gg w_{1}$ because $r_{\text {loss }}$ is mainly determined by the mass ratio, which was stopped by the small vertical wall of the protrusion, as will be explained later. Furthermore, since $X_{n}=\frac{H_{n}}{\sin \theta} \simeq \frac{\left(1-r_{\text {los }}\right)^{n}}{\sin \theta} H_{0}$, the observable travel length becomes small, as $\theta$ increases. In other words, if $\theta$ becomes too large, we cannot observe the side-shooting phenomenon appropriately. Therefore, in this experiment, we select $\theta=3^{\circ}$ as an appropriate condition.

\section{B. Energy loss ratio (Models A and B)}

We consider the masses $\left(m_{p 1}, m_{p 2}\right.$, and $\left.m_{p 3}\right)$ of the lower, middle, and upper parts, respectively, of the hemisphere with radius $r_{0}$, which have thicknesses of $l_{1}, l_{2}$, and $r_{0}-l_{1}-l_{2}$, as shown in Fig. 1(d). By assuming that the kinetic energy of the middle part is lost due to the impinging of the protrusion with thickness $l_{2}$ and that the kinetic energy of $m_{p 1}$ is converted into the surface energy of the new surface under the protrusion plate of area
$S_{w}\left(w, r_{0}, l_{1}\right)$, we obtain

$$
\begin{gathered}
r_{\text {loss }}^{\text {modelA }} \simeq m_{p 2} / m_{p}, \\
\gamma S_{w}\left(w, r_{0}, l_{1}\right) \simeq m_{p 1} g H_{0},
\end{gathered}
$$

where $S_{w}\left(w, r_{0}, l_{1}\right)=f_{a}\left(w, \sqrt{r_{0}^{2}-l_{1}^{2}}\right), w$ represents the push-in width, and the area function $f_{a}(w, r)\left(r=\sqrt{r_{0}^{2}-l_{1}^{2}}\right)$ is given by $f_{a}(w, r)=r^{2} \cos ^{-1}\left(1-\frac{w}{r}\right)-(r-w) \sqrt{w(2 r-w)}$. We call this model as Model $\mathrm{A}$, and it is useful under the condition $w \ll l_{3}$, whereas under the condition $w \sim l_{3}$, we need to consider the energy loss of $m_{p 1}$ as Model B. Namely, we obtain

$$
r_{\text {loss }}^{\text {modelB }} \simeq \frac{m_{p 2}+r_{B} m_{p 1}}{m_{p}}
$$

where $r_{B}\left(0 \leq r_{b} \leq 1\right)$ is the loss ratio of the kinetic energy of $m_{p 1}$. Here, $m_{p 1}=\rho\left[f_{v}\left(r_{0}, r_{0}\right)-f_{v}\left(r_{0}, r_{0}-l_{1}\right)\right], m_{p 2}=\rho\left[f_{v}\left(r_{0}, r_{0}-l_{1}\right)-\right.$ $\left.f_{v}\left(r_{0}, r_{0}-l_{1}-l_{2}\right)\right]$, and the volume function $f_{v}(r, h)$ is given by $f_{v}(r, h)=\frac{\pi}{6} h\left[3 h(2 r-h)+h^{2}\right]$. Furthermore, we determined $r_{B}\left(X_{n}\right)$ as $r_{B}\left(X_{n}\right) \simeq 0.8\left(X_{n} / X_{0 c}\right)^{4}$ to explain our experimental results [which will be explained in Fig. 6(b)], where $X_{0 c}=5 \mathrm{~cm}$; however, since $r_{B} \leq 1$, we set $r_{B}\left(X_{n}\right) \simeq 0.8\left(X_{n} / X_{0 c}\right)^{4}$ at $X_{n}<5.29 \mathrm{~cm}$ and $r_{B}=1$ at $X_{n} \geq 5.29 \mathrm{~cm}$, in Eq. (5). Note that if $w \sim l_{3}, r_{\text {loss }}$ is not constant in the sense that $r_{B}\left(X_{n}\right)$ changes for every impinging event. Furthermore, at the moment of impact, the liquid can transfer some momentum in the lateral directions. However, the kinetic energy in the lateral directions is preserved and converted into the surface energy until the motion of the droplet is stopped. Therefore, the transfer of momentum in the lateral directions needs not be considered in the current problem.

\section{Fluctuation model to account for vertical vibration (Model C)}

We consider the effect of fluctuation of $h_{1}$ [in Fig. 1(b)] due to a vertical vibration of the droplet. Since impinging volume decreases as $h_{1} / r_{0}$ increases, the prediction of $r_{\text {loss }}$ is simply corrected as follows:

$$
r_{\text {loss }}^{\text {modelc }}\left(h_{1}, r_{0}\right) \simeq \frac{r_{0}}{h_{1}} \frac{m_{p 2}+r_{B} m_{p 1}}{m_{p}} .
$$

Note that since we observed a relatively large variation of $X_{1}$ in the experiment, we need to consider this model (Model C) to recognize the cause, and Eq. (6) successfully explains the fluctuation, as will be presented in Figs. 5(a) and 5(b). Here, since the height of the droplet extends $\frac{h_{1}}{r_{0}}$ times in the vertical direction, the length of the middle part of $m_{p 2}$ also extends $\frac{h_{1}}{r_{0}}$ times; i.e., the deformed length of the middle part of $m_{p 2}$ is written as $l_{2}^{\prime}=\frac{h_{1}}{r_{0}} l_{2}$. Similarly, the deformed length of the lower part of $m_{p 1}$ is written as $l_{1}^{\prime}=\frac{h_{1}}{r_{0}} l_{1}$. Since the ratio of the impinging mass $m_{p 2}^{\prime}$ to the mass $m_{p 2}$ of the deformed part is in the ratio of $l_{2}$ to $l_{2}^{\prime}$, we can write $\frac{m_{p 2}^{\prime}}{m_{p 2}}=\frac{l_{2}}{l_{\frac{1}{1}}}=\frac{r_{0}}{h_{1}}$. Similarly, we can write $\frac{m_{p 1}^{\prime}}{m_{p 1}}=\frac{l_{1}}{l_{1} \frac{h_{1}}{r_{0}}}=\frac{r_{0}}{h_{1}}$. Thus, for the deformed particle, we can write $r_{\text {loss }}^{\text {modelC }}\left(h_{1}, r_{0}\right) \simeq \frac{m_{p 2}^{\prime}+r_{B} m_{p 1}^{\prime}}{m_{p}} \simeq \frac{r_{0}}{h_{1}} \frac{m_{p 2}+r_{B} m_{p 1}}{m_{p}}$. 


\section{Time model (Model D)}

We consider the Lagrange equation of motion during the surface formation process at $t_{0} \leq t^{\prime} \leq t_{1}$; i.e.,

$$
\frac{d}{d t^{\prime}}\left(\frac{\partial L}{\partial \dot{X}^{\prime}}\right)-\frac{\partial L}{\partial X^{\prime}}=0
$$

where $L(=T-U)$ is the Lagrange function, $T\left(=\frac{1}{2} m_{p 1} \dot{X}^{\prime}{ }^{2}\right)$ is the kinetic energy of $m_{p 1}, U\left(=2 r A r_{0} \gamma X^{\prime}\right)$ is the potential energy due to the new surface formation, $\dot{X}^{\prime}=\frac{d X^{\prime}}{d t^{\prime}}$, and the direction of the $X^{\prime}$ axis is opposite to that of the $X$ axis. Note that since Eq. (4) is slightly inconvenient, we approximate $S_{w}\left(w, r_{0}, l_{1}\right) \sim 2 A r_{0} w$. Thus, we obtain

$$
w^{\text {ModelD }} \sim \frac{m_{p 1} g H_{0}}{2 A r_{0} \gamma}
$$

where $A(=0.83)$ is the adjustment parameter, which is determined as $A \simeq 0.83$ from the experimental results [in Fig. 6(a)]. From Eq. (7), we obtain

$$
m_{p 1} \ddot{X}^{\prime}=-2 A r_{0} \gamma
$$

Thus, by solving Eq. (9) under the initial condition that $\dot{X}^{\prime}=V_{0}=\sqrt{2 g H_{0}}$ and $X^{\prime}=0$ at $t^{\prime}=0$, we obtain

$$
\dot{X}^{\prime}=\sqrt{2 g H_{0}}-\frac{2 A r_{0} \gamma}{m_{p 1}} t^{\prime}
$$

Hence, by considering $\dot{X}^{\prime}=0$, we obtain a theoretical push-in time as

$$
\Delta t_{w}^{\text {modelD }}=\frac{m_{p 1}}{2 A r_{0} \gamma} \sqrt{2 g H_{0}} .
$$

\section{EXPERIMENTAL RESULTS}

\section{A. Launching effect}

Figure 3 shows the launching effect of the side-shooter. In Fig. 3, the symbols and lines show the experimental and theoretical results, respectively. As shown in Figs. 3(a)-3(c), the droplet was experimentally launched to the level of $X_{0} \simeq 1.5-3 \mathrm{~cm}$ by the sideshooter, and our prediction using Model $\mathrm{C}$ with an average value of $\overline{h_{1}} / r_{0}=1.29$ explains the observed launching height to some extent, although significant fluctuation exists, as will be discussed later in detail. Figure 3(d) shows the dependence of the velocity $V=\frac{d X}{d t}$ on $t$, while Fig. 3(e) shows the velocity $V_{p}$ corresponding to $X_{p}$ (or $H_{p}$ ). Here, $V_{p}=\sqrt{2 g X_{p} \sin \theta}(p=1,2,3)$ shows the launching velocity calculated from $X_{p}$. In Fig. 3(e), the theoretical curve of Model $\mathrm{C}^{\prime}$ is obtained from $V_{p}\left(N_{p}\right)=\sqrt{2 g\left(1-r_{\text {loss }}\right)^{N_{p}} X_{0} \sin \theta}$ under the assumption that $r_{\text {loss }}=r_{\text {loss }}^{\text {model } C}=0.603$ with $r_{B}=r_{B}\left(X_{0}\right)=0.898$ in Eq. (6), whereas the theoretical dots $(x)$ of Model $C$ are calculated from $V_{p}\left(N_{p}\right)=\sqrt{2 g\left(1-r_{\text {loss }}^{\text {model } C}\right) X_{N_{p}-1} \sin \theta}$ under the assumption that $r_{\text {loss }}^{\text {model } C}=r_{\text {loss }}^{\text {model } C}\left(X_{N_{p}-1}\right)$; e.g., $r_{\text {loss }}^{\text {model } C}=0.603,0.295$, and 0.285 $\left(r_{B}=0.898,0.317\right.$, and 0.007$)$ for $N_{p}=1,2$, and 3 , respectively.
Although the droplet volume decreases gradually with respect to time, the effect seems to be negligible for small $N_{p}$ values, since the theoretical plot using Model $\mathrm{C}$ agrees with the experimental $V_{p}$ in Fig. 3(e). From Figs. 3(d) and 3(e), we find that the maximum launching velocity is approximately $15 \mathrm{~cm} / \mathrm{s}$, and it is comparable to that of the liquid delivery system of Linke et al., ${ }^{5}$ which is much higher than that of other systems. Thus, the performance of our side-shooter is outstanding in spite of the simple structure; e.g., it does not require a sawtooth-like structure on the slope and just needs a hot protrusion. Figure $3(\mathrm{f})$ shows the experimental trajectory $\left(x_{p}(t), y_{p}(t)\right)$ of the droplet, which was obtained by using the image processing technique; the (red) dots in Fig. 3(f) represent a real droplet trajectory and not a conceptual trajectory. As shown in Fig. 3(f), we can control the trajectory of the droplet freely in a twodimensional (2D) plane by using the reflection effect of the sideshooters [which is represented by the three rectangular plates in Fig. 3(f)]. In other words, by using multiple side-shooters, we can change the direction of the droplet freely in a $2 \mathrm{D}$ plane and design the trajectories at will. Thus, our side-shooter is useful for various microfluidic and biomedical applications. Since we can write that $V_{x}^{\text {out }}=V_{y}^{\text {in }}$ [from the momentum conservation in the parallel direction ( $x^{\prime}$ direction) to the protrusion plate of the side shooter] and $\quad V_{y}^{\text {out }}=\sqrt{2 g H_{\text {out }}}=\sqrt{2 g\left(1-r_{\text {loss }}\right) H_{\text {in }}}=\sqrt{\left(1-r_{\text {loss }}\right)} V_{y}^{\text {in }}$ [from Eqs. (1) and (2)], the incident and emergence angles are given by $\theta_{\text {in }}=\tan ^{-1} \frac{V_{y}^{\text {lin }}}{V_{x}^{\text {lin }}}$ and $\theta_{\text {out }}=\tan ^{-1} \frac{V_{y}^{\text {tout }}}{V_{x}^{\text {lout }}}=\tan ^{-1} \frac{V_{y}^{\text {lin }}}{V_{x}^{\text {lin }}} \sqrt{\left(1-r_{\text {loss }}\right)}$, respectively, where $\left(V_{x}^{\text {in }}, V_{y}^{\text {/in }}\right)$ and $\left(V_{x}^{\text {out }}, V_{y}^{\text {out }}\right)$ are the incident and emergence velocities, respectively. In other words, the reflection effect of the side-shooter is a phenomenon caused by the hot protrusion (which reflects the droplet due to the launching effect of the side-shooter).

\section{B. Surface formation process due to the Leidenfrost phenomenon}

Figure 4 shows photographs of the surface formation process due to the new Leidenfrost phenomenon at $t=t_{0}, t_{w 1}, t_{w 2}$, and $t_{1}$ for $N=1$ and 2. In Figs. 4(a) and 4(e), $h_{1}=4.23$ and $3.53 \mathrm{~mm}$, respectively; i.e., $h_{1}$ was significantly different in each experiment. This is because there exists a vertical vibration in the droplet, which was already explained in detail by using numerical simulations in Ref. 23, and this is the reason for the fluctuation of $X_{p}$. Interestingly, the event of higher $h_{1}(4.23 \mathrm{~mm})$ at $N=1$ results in a larger $w_{1}$ value $\left(w_{1}>w_{2}\right)$, whereas the event of lower $h_{1}(3.53 \mathrm{~mm})$ at $N=2$ results in a larger $w_{2}$ value $\left(w_{2}>w_{1}\right)$, as shown in Figs. 4(c) and 4(g), respectively. This is probably because the droplet of lower $h_{1}$ extends in the upper direction in the subsequent period, whereas the droplet of higher $h_{1}$ shrinks in the lower direction in the subsequent period. Furthermore, as shown in Figs. $4(\mathrm{~b})$ and $4(\mathrm{f})$, the kinetic energy of $m_{p 2}$ (and a part of $m_{p 1}$ ) is lost due to the collision with the hot protrusion plate of thickness $l_{2}=1 \mathrm{~mm}$, as discussed in Sec. IV B. Although the entire energy is usually lost due to the collision in ordinary fluid dynamic systems (as pointed out before), the remaining energy is preserved as the surface energy in the side-shooter because of the formation of a new surface due to the folded Leidenfrost vapor layer resulting from the hot 


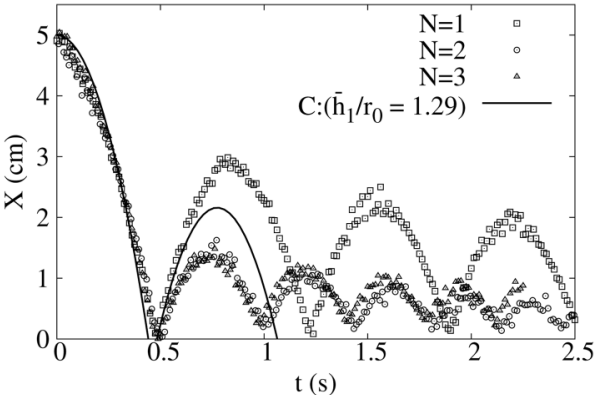

(a)

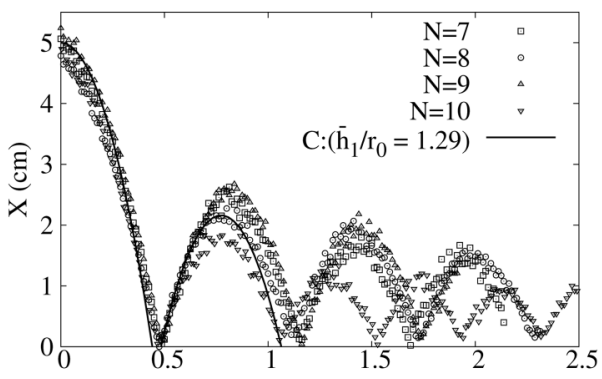

$\mathrm{t}(\mathrm{s})$

(c)

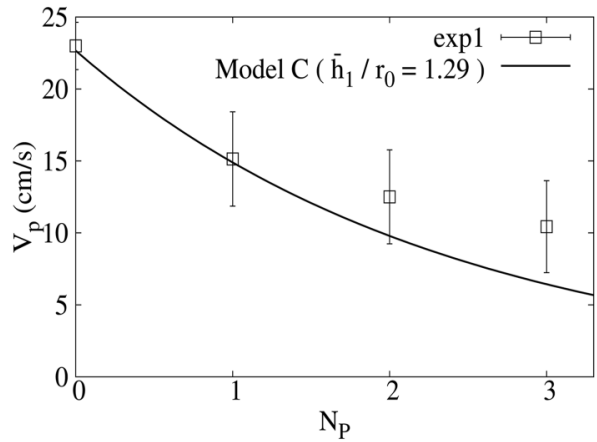

(e)

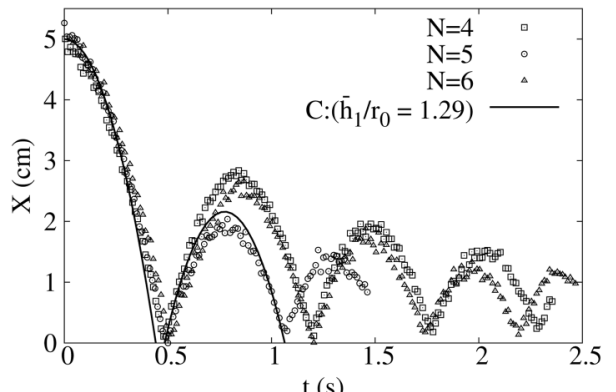

(b)

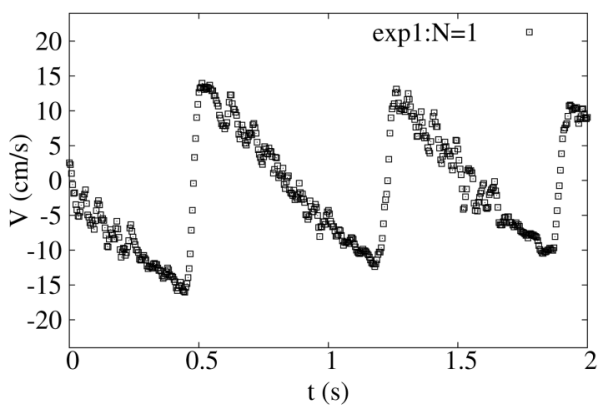

(d)

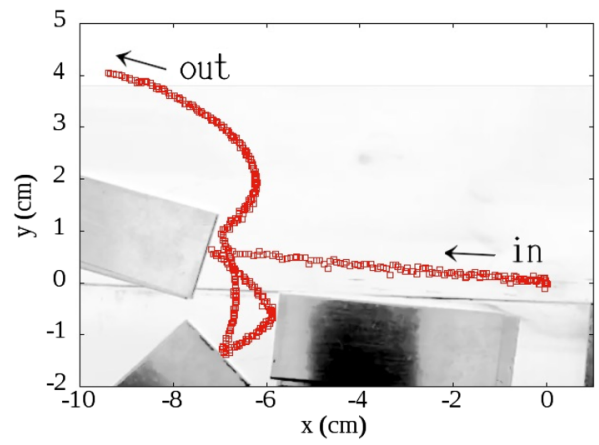

(f)
FIG. 3. Launching effect of the sideshooter. (a) $X$ vs $t(N=1-3)$. (b) $X$ vs $t(N=4-6)$. (c) $X$ vs $t(N=7-10)$. (d) $V$ vs $t(N=1)$. (e) $V$ vs $N_{p}$. (f) $2 \mathrm{D}$ shooting phenomenon due to the reflection phenomenon of the sideshooter. Here, $T_{s}=300^{\circ} \mathrm{C}, \theta=3^{\circ}$, $\phi=5 \mathrm{~mm}, r_{0}=3.15 \mathrm{~mm}, X_{0}=5 \mathrm{~cm}$, $I_{1}=I_{2}=1 \mathrm{~mm}$, and $l_{3}=2.5 \mathrm{~mm}$. protrusion plate, and the accumulated surface energy is used for launching the droplet, as shown in Figs. 4(d) and 4(h).

\section{Fluctuations due to $\mathrm{h}_{1}$}

Figure 5 shows the fluctuations due to $h_{1}$ for two data sets (i.e., Experiments 1 and 2; $N_{t}=10$ for each experiment), which were obtained by the same experiment under the same condition on different dates. As shown experimentally in Figs. 5(a) and 5(c), as $h_{1} / r_{0}$ increases, the dependence of $H_{1}$ increases, while the energy loss ratio $E_{\text {loss }} / E_{\text {in }}=\left(H_{0}-H_{1}\right) / H_{0}$ decreases; these experimental results are theoretically explained fairly well by Model C, although there are exceptions. For example, the data set of $h_{1} / r_{0}=$ 1.42 and $H_{1}=0.780 \mathrm{~mm}$ in Fig. 5(a) for $N=3$ (in Experiment 1) is an exception. However, we consider that such exceptionally low values of $H_{1}$ occur when $w_{2} \gg w_{1}\left(w_{1}=3.69 \mathrm{~mm}, w_{2}=4.62 \mathrm{~mm}\right.$ for $N=3$ ); i.e., probably because of the fluctuation of the internal flow condition due to the vertical vibration, $w_{2}$ becomes much larger than $w_{1}$, and in that case, the remaining kinetic energy is not effectively converted into surface energy. Furthermore, Figs. 5(c) and 5(d) show the dependences of $w$ and $\Delta t_{w}$ on $h_{1}$. Unfortunately, we could not find a clear trend in them. However, in Fig. 5(c), it can be seen that $w_{1}$ is limited by $l_{3}=2.5 \mathrm{~mm}$, as is considered in Model $\mathrm{B}$, whereas the order of $\Delta t_{w}$ is $20 \mathrm{~ms}$ as predicted by Model D.

\section{Dependences on $X_{0}$}

Figure 6 shows the dependences on $X_{0}$. In Fig. 6, the symbols show the experimental results, whereas the lines show the 


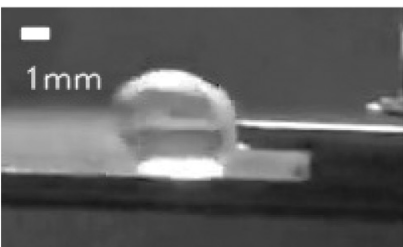

(a)

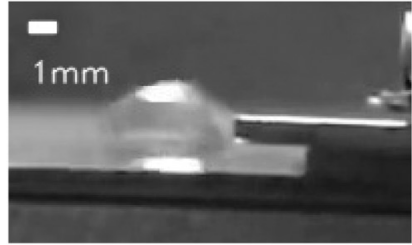

(e)

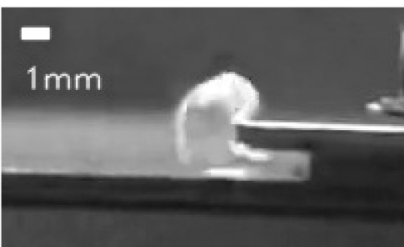

(b)

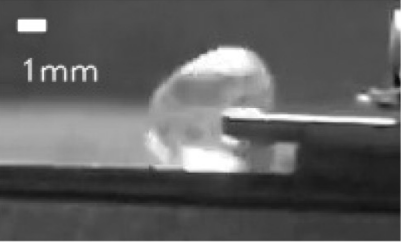

(f)

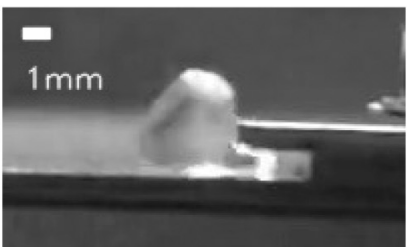

(c)

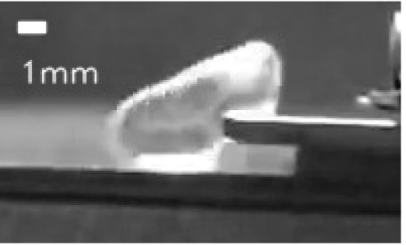

(g)

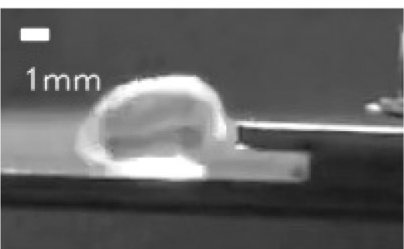

(d)

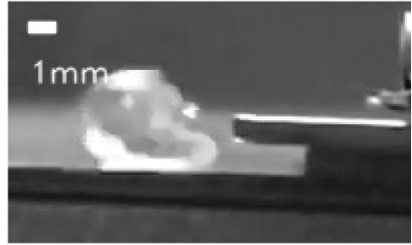

(h)

FIG. 4. Surface formation process due to a new Leidenfrost phenomenon. (a) $t=t_{0}(N=1)$. (b) $t=t_{w 2}(N=1)$. (c) $t=t_{w 1}(N=1)$. (d) $t=t_{1}$ ( $\left.N=1\right)$. (e) $t=t_{0}$ $(N=2)$. (f) $t=t_{\mathrm{w} 1}(N=2)$. (g) $t=t_{\mathrm{w} 2}(N=2)$. (h) $t=t_{1}(N=2)$. Here, $m_{p}=6.26 \times 10^{-5} \mathrm{~kg}, \gamma=58.9 \mathrm{mN} / \mathrm{m}$ at $T_{b}=100^{\circ} \mathrm{C}, T_{s}=300^{\circ} \mathrm{C}, \theta=3^{\circ}, \phi=5 \mathrm{~mm}$, $r_{0}=3.15 \mathrm{~mm}, X_{0}=5 \mathrm{~cm}, l_{1}=l_{2}=1 \mathrm{~mm}$, and $l_{3}=2.5 \mathrm{~mm}$; for $N=1(N=2), \Delta t_{\mathrm{w} 1}=0.0029 \mathrm{~s}(0.0250 \mathrm{~s})$ and $\Delta t_{\mathrm{w} 2}=0.0166 \mathrm{~s}(0.0375 \mathrm{~s})$.

theoretical results. As shown in Figs. 6(a)-6(c), the experimentalresults of the dependences of $w, E_{\text {loss }}$, and $H_{1}$ on $X_{0}$ agree with the theoretical results fairly well. Specifically, in the experiment, $w$ increases with increase in $X_{0}$, and this trend (especially for $w_{1}$ ) is in good agreement with the theoretical prediction by Model $\mathrm{A}$, which is intrinsically the model for $w_{1}$, although $w_{1}$ at $X_{0}=5 \mathrm{~cm}$ is experimentally limited by $l_{3}=2.5 \mathrm{~mm}$. Furthermore, the energy loss ratio $E_{\text {loss }} / E_{\text {in }}\left[=\left(H_{0}-H_{1}\right) / H_{0}\right]$ increases with increase in $X_{0}$, and this trend is successfully explained by Model B. In addition, the dependence of $H_{1}$ on $X_{0}$ has a peak at $X_{0}=4 \mathrm{~cm}$, and this

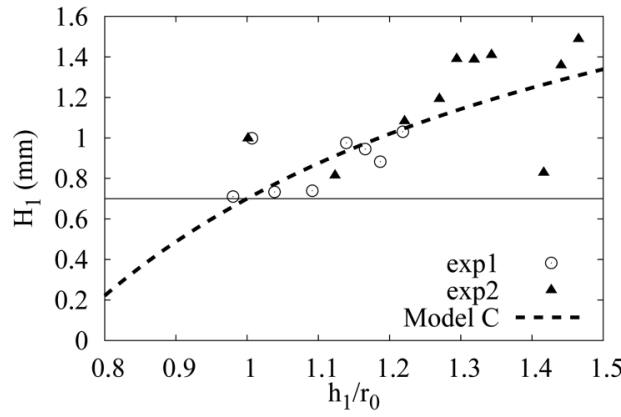

(a)

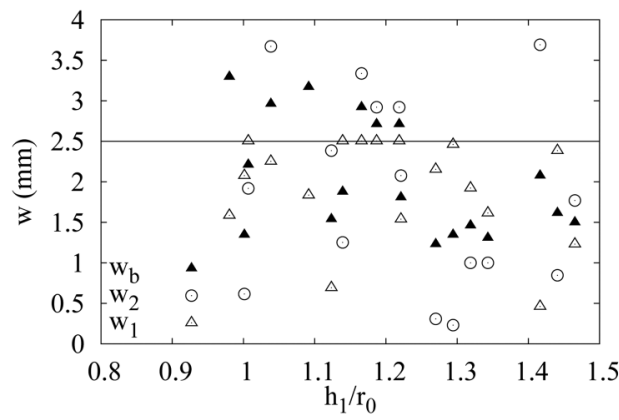

(c)

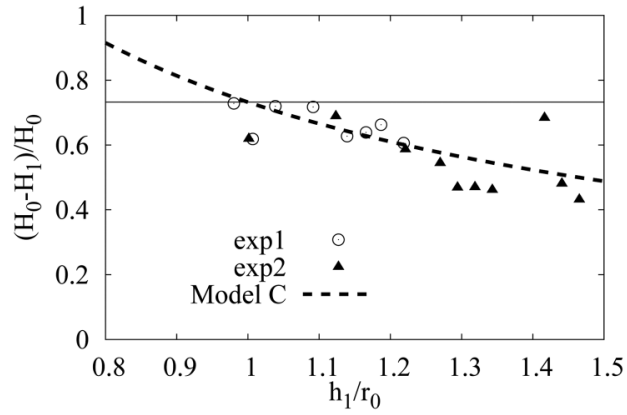

(b)

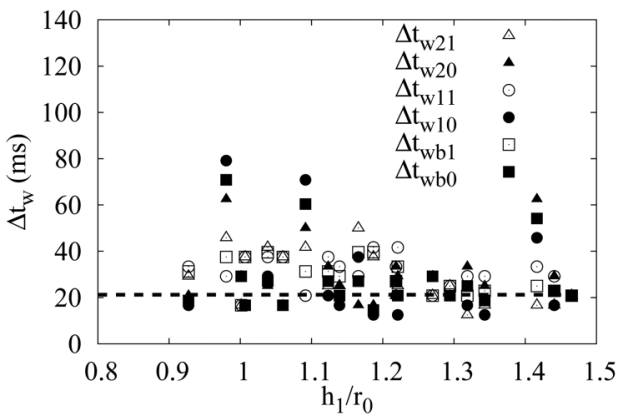

(d)
FIG. 5. Fluctuations due to $h_{1}$. (a) $H_{1}$ vs $h_{1}$. (b) $E_{\text {loss }}$ vs $h_{1}$. (c) $w$ vs $h_{1}$. (d) $\Delta t_{w}$ vs $h_{1}$. Here, $m_{p}=6.26 \times 10^{-5} \mathrm{~kg}$, $\gamma=58.9 \mathrm{mN} / \mathrm{m}$ at $T_{b}=100^{\circ} \mathrm{C}$, $T_{s}=300^{\circ} \mathrm{C}, \quad \theta=3^{\circ}, \quad \phi=5 \mathrm{~mm}$, $r_{0}=3.15 \mathrm{~mm}, \quad X_{0}=5 \mathrm{~cm}, l_{1}=l_{2}=$ $1 \mathrm{~mm}$, and $l_{3}=2.5 \mathrm{~mm}$. In (a) and (b), the solid lines show the average values using Model $\mathrm{C}$. In (c), the solid line shows the limitation of $w_{1}$ due to $l_{3}=2.5 \mathrm{~mm}$. In (d), the broken line shows the theoretical result using Model D. 


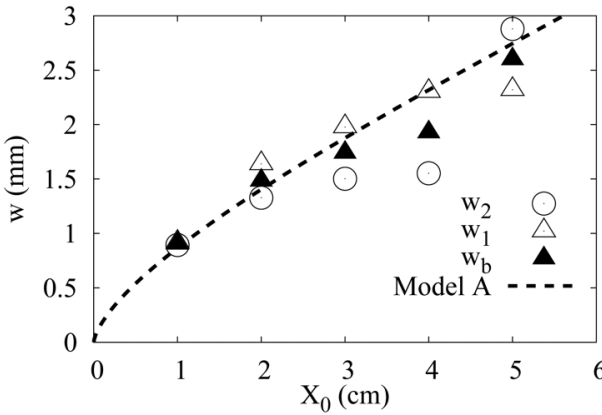

(a)

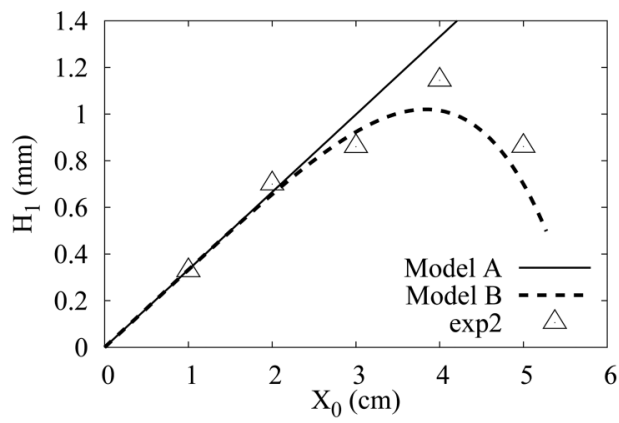

(c)

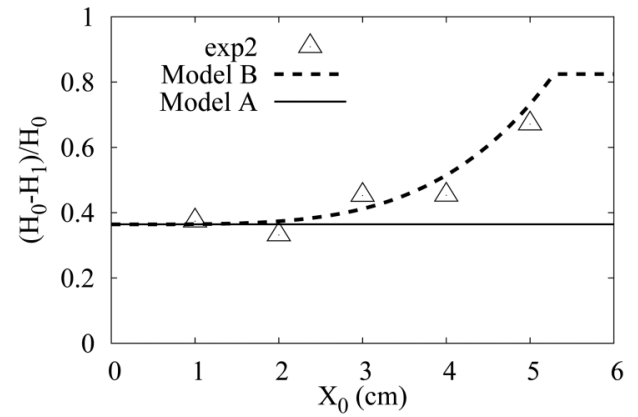

(b)

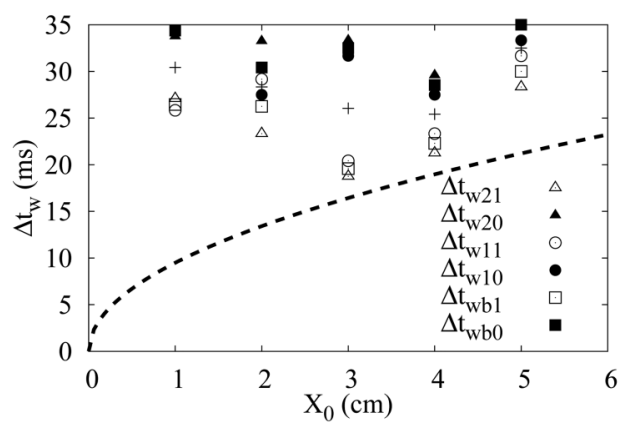

(d)
FIG. 6. Dependences on $X_{0}$. (a) $w$ vs $X_{0} \quad\left(I_{3}=1 \mathrm{~mm}\right)$. (b) $E_{\text {loss }}$ vs $X_{0}$ $(\phi=5 \mathrm{~mm})$. (c) $H_{1}$ vs $X_{0}(\phi=5 \mathrm{~mm})$. (d) $\Delta t_{w}$ vs $X_{0}(\phi=5 \mathrm{~mm})$. Here, $m_{p}=6.26 \times 10^{-5} \mathrm{~kg}, \gamma=58.9 \mathrm{mN} / \mathrm{m}$ at $T_{b}=100^{\circ} \mathrm{C}, T_{s}=300^{\circ} \mathrm{C}, \theta=3^{\circ}$, $\phi=5 \mathrm{~mm}, \quad r_{0}=3.15 \mathrm{~mm}, \quad l_{1}=l_{2}=$ $1 \mathrm{~mm}$, and $l_{3}=2.5 \mathrm{~mm}$; the symbols show the experimental results, whereas the lines show the theoretical results. trend is also successfully explained by Model B. Thus, we consider that our simple model is very useful, and the physics of the sideshooting phenomenon is successfully described by our model. However, while the experimental results of the push-in time $\left(\Delta t_{w}\right)$ is approximately $20-30 \mathrm{~ms}$, our time model (Model D) just predicts the order at $X_{0} \geq 3 \mathrm{~cm}$. This is probably because the energy of the vertical vibration affects $\Delta t_{w}$, and this results in hiding the mean trend of the time model.

\section{DISCUSSION}

Although Linke et al..$^{5}$ and Ok et al. ${ }^{7}$ have already shown highspeed liquid delivery systems of $V \sim 5$ and $\sim 40 \mathrm{~cm} / \mathrm{s}$, respectively, by using an asymmetrical sawtooth-like structure at $T_{s, 1}>T_{L}$, we were the first to show that a side-shooter having a plate-like hot protrusion can shoot a water droplet with a velocity of $\sim 15 \mathrm{~cm} / \mathrm{s}$; we have also clarified the side-shooting mechanism. Our side-shooter can shoot the droplet with high velocity by using the accumulated surface energy of the liquid due to the formation of a new folded Leidenfrost vapor layer, which results from a new Leidenfrost phenomenon coupled with the protrusion at $T_{s, 1}>T_{L}$. This mechanism is very different from that of the device of Linke et al., since the liquid droplet (in the device of Linke et al.) is considered to be driven by the pressure-driven flow (due to $\nabla P)^{10}$ or the thermal creep (due to $\nabla T$ ). ${ }^{9}$

Moreover, the structure of our side-shooter is very simple; e.g., unlike the device of Linke et al., ${ }^{5}$ our side-shooter does not require sawtooth-like structures on the substrate. Furthermore, unlike the liquid delivery system using an electrowetting phenomenon, ${ }^{4}$ our side-shooter does not require a high-voltage matrix driver and matrix electrodes to apply a strong electric field. In addition, since our side-shooter has a reflection effect [as demonstrated in Fig. 3(f)], there is a possibility that we can realize promising biomedical systems by controlling the trajectories of the droplet at will in a $2 \mathrm{D}$ plane in the future. Furthermore, similar to the device of Linke et al., ${ }^{5}$ there is a possibility of developing a system that requires no power supply and operates at a high temperature. Furthermore, similar to the heat engine ${ }^{14}$ that uses the principle of the device of Linke et al., a new heat engine that uses our side-shooting mechanism can be realized in the future.

Although a large fluctuation was observed in the launching performance [presented in Figs. 3(a)-3(c)], we can probably suppress the fluctuation in the future, since we have already recognized that the vertical vibration of the droplet is the main cause of the fluctuation [as presented in Figs. 3(a) and 3(b)]. Specifically, the vibration perpendicular to the brass plate results from the nonzero value of the Weber number of the droplet $\left(W e=\frac{\rho \phi U_{v}^{2}}{\gamma}\right)$ at $X_{0}$, where $U_{v}$ is the velocity perpendicular to the brass plate at $X_{0}{ }^{23}$ Therefore, if we place the liquid at $X_{0}$ with $U_{v}=0$, we can suppress the fluctuation, since $W e \sim 0$. Although we tried minimizing the $W e$ number in our experiment, it was impossible to place the droplet on the brass plate without generating the vertical vibration, because we cannot remove the needle of the syringe from the droplet at a stable state. If we assume that we need the space $\Delta h(\sim 0.1 \mathrm{~mm})$ between the upper surface of the droplet and the tip of the needle, we can approximate that $U_{v} \sim \sqrt{2 g \Delta h} \sim 0.04 \mathrm{~m} / \mathrm{s}$ and $W e \sim 0.2$. Furthermore, if we can use a much smaller droplet in microfluidic 


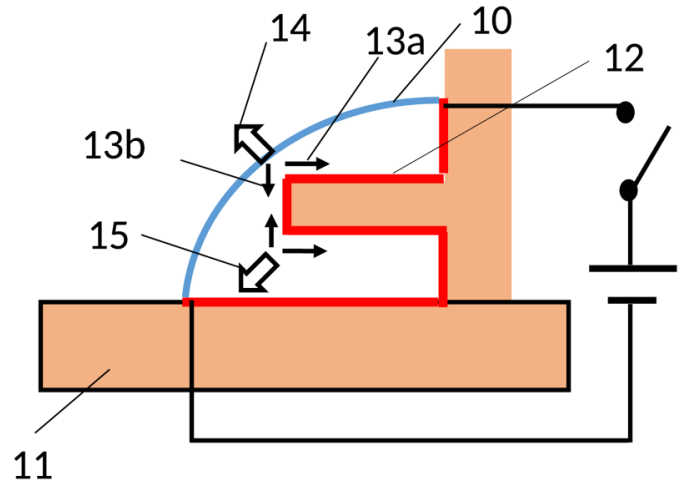

FIG. 7. Schematic view of an active side-shooter using a Leidenfrost phenomenon coupled with a protrusion having a heater. 10: water droplet, 11: substrate (hydrophobic), 12: thin-film heater (hydrophilic), 13a,13b: surface tension at the corner, 14: the net force that works on the droplet at the upper corner, 15: the net force that works on the droplet at the lower corner.

systems, we can also suppress the fluctuation, because We becomes small as $\phi$ becomes small.

In this paper, we have demonstrated a passive side-shooting system that relaunches a droplet falling onto a protrusion. However, our experimental result clearly shows that we can realize an active side-shooting system that launches a stationary droplet. For example, Fig. 7 shows an active side-shooter that uses a Leidenfrost phenomenon coupled with a protrusion having a heater. As shown in Fig. 7, the thin-film heater has a hydrophilic surface, whereas the substrate has a hydrophobic surface. Thus, we can set the water droplet on the thin-film heater and keep the droplet in a state of rest. Then, we suddenly heat up the droplet with a heat flux of approximately $1 \mathrm{GW} / \mathrm{m}^{2}$ by applying a sudden voltage. As a result, a deformed Leidenfrost vapor layer is generated along the heater. Hence, the surface tension denoted by $13 \mathrm{a}$ and $13 \mathrm{~b}$ works on the upper corner of the protrusion, and the net force denoted by 14 (based on Newton's third law) works on the droplet. Similarly, the net force denoted by 15 works on the droplet at the lower corner. Consequently, a net force of $\sim 2 \gamma \phi$ is generated, and the droplet is shot in the left direction with a velocity of $\sim 2 \gamma \phi \Delta t / m_{p}$, where $\Delta t$ is the time that the droplet is pushed out from the side-shooter. From Fig. 5(d), we can see that $\Delta t \sim \Delta t_{w} \sim 20 \mathrm{~ms}$, $\gamma \sim 60 \mathrm{mN} / \mathrm{m}, \phi \sim 5 \mathrm{~mm}$, and $m_{p} \sim 6 \times 10^{-5} \mathrm{~kg}$, and we obtain the velocity as $v_{p} \sim 2 \gamma \phi \Delta t / m_{p} \sim 0.1 \mathrm{~m} / \mathrm{s}$; i.e., we can realize an active side-shooter that shoots the droplet with a velocity of $\sim 0.1 \mathrm{~m} / \mathrm{s}$. Namely, the side-shooting phenomenon is applied to both active and passive side-shooters, and the side-shooting phenomenon is generally defined as an acceleration phenomenon caused by the deformed Leidenfrost vapor layer along the nonflat surface, which has a temperature above the Leidenfrost temperature. Note that a simple vertical hot wall cannot relaunch a droplet because the kinetic energy of the droplet is almost lost by the droplet-wall collision. (See the supplementary material for the videos.)

Since the side-shooting phenomenon is an acceleration phenomenon caused by the deformed Leidenfrost vapor layer, we can use this principle for various actuators in the future. Specifically, by setting several hot oblique protrusions on a water layer in a microfluidic channel, we can accelerate the water as a microfluidic pump. On the other hand, by floating a substrate with several hot protrusions in water, we can move the substrate as a microfluidic container that transports materials. Similarly, we can use this to drive a valve in a microfluidic channel. Here, we mainly consider an active side-shooting phenomenon. However, we can also use a passive-shooting phenomenon to design a new self-propelled motion in the future. For example, by using several hot protrusions that are set on a hot plate in the circumferential direction, we can rotate water as a microfluidic mixer, although we need more extensive studies in the future to realize it. That is, this principle can be applied to a wide range of applications in microfluidics.

\section{CONCLUSION}

In conclusion, (1) we observed that a side-shooter with a platelike hot protrusion launched a water droplet with a high speed ( $\sim 15 \mathrm{~cm} / \mathrm{s})$. (2) By proposing a simple model that considers the formation of a new surface due to a new Leidenfrost phenomenon coupled with a protrusion, we have successfully explained the sideshooting phenomenon. We believe that our side-shooter is useful in microfluidics.

\section{SUPPLEMENTARY MATERIAL}

See the supplementary material for the videos of the sideshooting phenomenon.

\section{ACKNOWLEDGMENTS}

This work was partially supported by JSPS KAKENHI (Grant No. JP16K05650).

\section{REFERENCES}

${ }^{1}$ T. Squires and S. Quake, Rev. Mod. Phys. 77, 977 (2005).

${ }^{2}$ S. Yao, A. M. Myers, J. D. Posner, K. A. Rose, and J. G. Santiago, J. Microelectromech. Syst. 15, 717 (2006).

${ }^{3}$ J. P. Urbanski, T. Thorsen, J. A. Levian, and M. Z. Bazant, Appl. Phys. Lett. 89, 143508 (2006).

${ }^{4}$ M. G. Pollack, R. B. Fair, and A. D. Shenderov, Appl. Phys. Lett. 77, 1725 (2000).

${ }^{\mathbf{5}}$ H. Linke, B. J. Alemán, L. D. Melling, M. J. Taormina, M. J. Francis, C. C. Dow-Hygelund, V. Narayanan, R. P. Taylor, and A. Stout, Phys. Rev. Lett. 96, 154502 (2006).

${ }^{6}$ D. Quéré, Annu. Rev. Fluid Mech. 45, 197 (2013).

${ }^{7}$ J. T. Ok, E. Lopez-Ona, D. E. Nikitopoulos, H. Wong, and S. Park, Microfluid. Nanofluidics 1, 1045-1054 (2011).

${ }^{8}$ T. R. Cousins, R. E. Goldstein, J. W. Jaworski, and A. I. Pesci, J. Fluid Mech. 696, 215-227 (2012).

${ }^{9}$ A. Würger, Phys. Rev. Lett. 107, 164502 (2011).

${ }^{10}$ S. Hardt, S. Tiwari, and T. Baier, Phys. Rev. E 87, 063015 (2013).

${ }^{11}$ Q. Li, Q. J. Kang, M. M. Francois, and A. J. Hu, Soft Matter 12, 302 (2016).

${ }^{12}$ A.-L. Biance, C. Clanet, and D. Quéré, Phys. Fluids 15, 1632 (2003).

${ }^{13}$ A. Hashmi, Y. Xu, B. Coder, P. A. Osborne, J. Spafford, G. E. Michael, G. Yu, and J. Xu, Sci. Rep. 2, 797 (2012). 
${ }^{14}$ G. G. Wells, R. Ledesma-Aguilar, G. McHale, and K. Sefiane, Nat. Commun. 6, 639 (2015).

${ }^{15}$ M. Shi, X. Ji, S. Feng, Q. Yang, T. J. Lu, and F. Xu, Sci. Rep. 6, 28574 (2016).

${ }^{\mathbf{1 6}} \mathrm{H}$. Sugioka and S. Segawa, AIP Adv. 8, 115209 (2018).

17. M. Arter, D. J. Cleaver, K. Takashina, and A. T. Rhead, Appl. Phys. Lett. 113, 243704 (2018).

${ }^{18}$ C. Luo, M. Mrinal, and X. Wang, Sci. Rep. 7, 12018 (2017).
${ }^{19} \mathrm{M}$. Mrinal, X. Wang, and C. Luo, Langmuir 33, 6307 (2017).

${ }^{20}$ J. T. Pham, M. Paven, S. Wooh, T. Kajiya, H.-J. Butt, and D. Vollmer, Nat. Commun. 8, 905 (2017).

${ }^{21}$ A. Bouillant, T. Mouterde, P. Bourrianne, A. Lagarde, C. Clanet, and D. Quéré, Nat. Phys. 14, 1188 (2018).

${ }^{\mathbf{2 2}}$ M. Auliano, M. Fernandino, P. Zhang, and C. A. Dorao, Int. J. Heat Mass Transf. 124, 307 (2018).

${ }^{23}$ Y. Ge and L.-S. Fan, Phys. Fluids 17, 027104 (2005). 